\title{
p16 immunostaining in histological grading of anal squamous intraepithelial lesions: a systematic review and meta-analysis
}

\author{
Andreia Albuquerque $\mathbb{( i d}^{1,2} \cdot$ Elisabete Rios ${ }^{2,3,4} \cdot$ Claudia Camila Dias ${ }^{5,6} \cdot$ Mayura Nathan ${ }^{1}{ }^{1}$
}

Received: 14 November 2017 / Revised: 12 January 2018 / Accepted: 12 January 2018 / Published online: 13 February 2018

(c) United States \& Canadian Academy of Pathology 2018

\begin{abstract}
p16 is the most widely studied biomarker in lower anogenital tract squamous intraepithelial lesions and, currently the only recommended biomarker for histological grade assessment. The aim of this systematic review and meta-analysis was to evaluate p16-positive rates according to anal squamous intraepithelial lesions/anal intraepithelial neoplasia (AIN) grade. Two investigators independently searched four electronic databases: PubMed, Web of Sciences, Scopus, and Embase from inception until August 2017. Studies that evaluated p16 immunostaining in histological samples of anal and/or perianal squamous intraepithelial lesions and defined a p16-positive result as diffuse block staining with nuclear or nuclear plus cytoplasmic staining were included. A meta-analysis was performed using a random effects model. Fifteen studies consisting of 790 samples were included. The proportion of p16 expression increased with the severity of histological grade. p16 positivity was $2 \%(95 \%$ CI: $0.2-5 \%$ ) in normal histology, $12 \%$ (95\% CI: 2-27\%) in low-grade squamous intraepithelial lesions (LSILs)/AIN1 (excluding condylomas), 7\% (95\% CI: 2-13\%) in all LSIL (AIN1/LSIL/condyloma), 76\% (95\% CI: 61-88\%) in AIN2, and 90\% (95\% CI: 82-95\%) in AIN3. For anal high-grade squamous intraepithelial lesions (HSILs), in studies using a two-tiered nomenclature, p16 positivity was 84\% (95\% CI: 66-96\%) and for all HSIL (AIN2, AIN3, HSIL combined) it was $82 \%$ (95\% CI: 72-91\%). In summary, p16 positivity in anal squamous intraepithelial lesions appears to be in a similar range to the commonly described cervical squamous intraepithelial lesions, however, for anal low-grade lesions positivity seems to be lower.
\end{abstract}

Electronic supplementary material The online version of this article (https://doi.org/10.1038/s41379-018-0026-6) contains supplementary material, which is available to authorized users.

Andreia Albuquerque

a.albuquerque.dias@gmail.com

1 Homerton Anal Neoplasia Service (HANS), Homerton University Hospital, London, UK

2 Faculty of Medicine of the University of Porto, Porto, Portugal

3 Department of Pathology, Centro Hospitalar São João, Porto, Portugal

4 Institute of Molecular Pathology and Immunology of the University of Porto (IPATIMUP), Porto, Portugal

5 Department of Community Medicine, Information and Health Decision Sciences, Faculty of Medicine of the University of Porto, Porto, Portugal

6 Center for Health Technology and Services ResearchCINTESIS, Porto, Portugal

\section{Introduction}

The most widely studied biomarker in the lower anogenital tract squamous lesions is p16 and most of the studies analyzed the relationship between p16-positive rates and histological grading. p16 is a tumor-suppressor protein that acts like a cyclin-dependent kinase inhibitor in the transition from the $\mathrm{G} 1$ to the $\mathrm{S}$ phase of the cell cycle. It binds to cyclin-dependent kinases 4 and 6 and maintains the retinoblastoma gene product in its hypophosphorylated state [1], which in turn binds to E2F transcription factor and prevents cell cycle progression. In human papillomavirus (HPV)-related tumors, E7 oncoprotein functionally inactivates retinoblastoma protein $[1,2]$. This inactivation is thought to have a negative feedback on intracellular levels of p16 leading to accumulation that can be detected by immunostaining [1].

In 2012, the lower anogenital squamous terminology (LAST) was published and recommended a two-tiered nomenclature for noninvasive HPV-associated lesions of the lower anogenital tract, including the terms low-grade 
squamous intraepithelial lesions (LSILs) and high-grade squamous intraepithelial lesions (HSILs) to replace the previous three-tiered system (intraepithelial neoplasia: -IN1, -IN2, -IN3). p16 has been recommended as the biomarker for histological grading, to be used in four possible situations: (1) differential diagnosis between precancer and a mimic of precancer, (2) in cases of -IN2, (3) interobserver disagreement in the interpretation of a precancerous lesion, and (4) high-risk referral situations (like cytology suggestive of HSIL with negative or low-grade biopsies) [3].

The importance of this biomarker is not only related to the grading of these lesions, but also for clinical decisions that rely on p16 results, as in the cases of -IN2 lesions. These lesions downgraded by a negative result are normally not treated, whereas p16-positive lesions (considered therefore HSIL) are thought to have a higher potential for progression to carcinoma and hence treated.

Data on p16 immunostaining in lower anogenital tract squamous lesions are mostly based on cervical studies and are largely generalized to other anogenital sites, like the anus. In 2009, a meta-analysis was published exclusively on cervical squamous intraepithelial lesions [4] and the definition of a positive result used differed from the more recent LAST classification definition [3]. There is a marked heterogeneity for what is considered a positive result, especially in "older" cervical studies, thus affecting the accuracy of the expression rates. There is a fundamental need for accurate data on this biomarker in anogenital neoplasia other than cervical disease, especially after the current indications for using it and the standardized definition for positivity in histological samples [3]. Studies that identify anal precancerous lesions that are more likely to progress to carcinoma are needed, given the predicted rise in incidence and mortality for anal cancer over the next two decades [5].

Our aim was to evaluate p16-positive rates in anal squamous intraepithelial lesions (ASILs) according to histological grade.

\section{Materials and methods}

\section{Search strategy, selection criteria, and outcome}

Four electronic databases were searched: PubMed, Web of Sciences, Scopus, and Embase for articles that were published from their inception until August 2017. The search was conducted by two investigators (AA and ER) and in case of any discrepancies a consensus was reached, and no disagreements required adjudication. The terms "anal carcinoma in situ", "anus neoplasm", "anal intraepithelial neoplasia", "anal squamous intraepithelial lesions", "anal squamous intraepithelial neoplasm", "anal squamous neoplasia", "anal dysplasia”, “anal precancer", “anogenital lesions", "anal squamocolumnar junction", and "p16" were used. We also reviewed reference lists of retrieved articles to identify other relevant studies.

All retrospective or prospective studies that evaluated p16 immunostaining in anal and/or perianal squamous intraepithelial lesions/anal intraepithelial neoplasia (AIN) histological samples and clearly described a positive result when there was a diffuse block staining with nuclear or nuclear plus cytoplasmic staining were included. Only articles written in English and with p16 immunohistochemistry assessment were included. The studies also needed to have a clear description of the number of cases per histological grade, the number of p16 positive/negative per grade, and a report of anal samples separately (when other anogenital lesions were also present).

Studies that considered a cytoplasmic only staining as a positive result (or exclusion was not clear), immunostaining in anal cytological samples or describe simultaneously p16 and Ki-67 results were excluded. Studies published only in an abstract form, case reports, evaluation of p16 expression only in anal squamous cell carcinoma (without an evaluation in ASIL) were also not included in this analysis.

Eight authors were contacted by email for further details. Two authors provided further information concerning sample size and expression for different histological grades [6] and for the definition of a positive result consistent with a diffuse block with nuclear plus cytoplasmic staining [7] and were included. Four other studies (three authors) were excluded, because cytoplasmic only staining was considered also a positive result $[8,9]$ or a clear confirmation of the exclusion of cytoplasmic only staining as a positive result was not obtained $[10,11]$. Three other authors that were contacted due to insufficient/incomplete data for analysis [12, 13] and a lack of clear definition of a positive result [14] did not respond, and the studies were not included.

In one study [15], authors considered as a positive result cytoplasmic and/or nuclear immunoreactivity, but in the results section a positive result was described as cytoplasmic and nuclear, and so the study was included.

This meta-analysis included studies published before and after the LAST consensus [3], so different nomenclatures are present according to the date of the study publication. ASIL and AIN, LSIL and AIN1, HSIL and AIN2/3 were used synonymously; the classification used in each study was adopted.

For low-grade lesions, three categories were described: condyloma acuminate only (as defined by the original study), AIN1/LSIL (anal condylomas excluded or there were no anal condylomas described), and all LSIL (LSIL, condyloma, AIN1, AIN1 and condyloma described together and condyloma with AIN1/LSIL). This last category 
corresponded to the definition of LSIL by LAST [3] (twotiered nomenclature). For high-grade lesions, four categories were described and analyzed according to the studies description: AIN2, AIN3, HSIL (as defined by LAST [3] in a two-tiered nomenclature), and all the combined HSIL (AIN2, AIN3 and HSIL).

Our outcome was the proportion of p16 positivity according to the histological grade of ASIL/AIN.

\section{Statistical analysis}

The pooled proportions and 95\% confidence intervals (CIs) for p16-positive immunostaining per histological grade were computed, using the proportions described in the original studies, and the random effects model, weighted for the sample size of each study, was considered the most appropriate for the analysis. Heterogeneity between studies was tested using $\mathrm{I}^{2}$ statistic with a $95 \%$ CI. For publication bias, funnel plots were derived for each histological grade. Statistic data analysis was performed using MedCalc Software version 17.5.3.

\section{Results}

The electronic search retrieved 207 studies of which 171 were excluded: 101 after review of the title, 5 after review of the abstract, and 65 were duplicated. In total, 36 were potentially eligible and subsequently scrutinized in full text. For the meta-analysis, 15 studies fulfilled the study criteria and were included (Fig. 1). There was a total of 790 samples in which p16 was performed and the results reported, and therefore could be considered for the analysis. For these studies, a descriptive table with the study characteristics was done, including the first author, country, and publication year, p16 positivity definition, antibodies used, total sample size, sample size for each histological grade, and p16 positivity in each of the histological grade (Table 1).

All studies included were observational. Most of them were conducted in USA (10 studies) [6, 7, 15-22], two were performed in Germany [23, 24], and one study each in Spain [25], Portugal [26], and Ireland [27]. Six did not describe the population from which ASIL samples were obtained [7, 16-19, 22]. One study included only inflammatory bowel disease patients [21] and another included only hemorrhoidectomy specimens [6].

The proportion of p16 expression increased with the severity of histological grade. On average 2\% (95\% CI: $0.2-5 \%$ ) of the normal biopsies, $12 \%$ (95\% CI: $2-27 \%$ ) of AIN1/LSIL (excluding condylomas), 7\% (95\% CI: 2-13\%) of all LSIL (AIN1/LSIL/condyloma), $76 \%$ (95\% CI: $61-88 \%)$ of AIN2, and $90 \%$ (95\% CI: $82-95 \%$ ) of AIN3 showed diffuse block nuclear or nuclear and cytoplasmic staining. For HSIL results, when studies considered a two-tiered nomenclature, the positive rate was 84\% (95\% CI: 66-96\%). For all HSIL results (all classified as AIN2, AIN3, or HSIL), p16 was positive in $82 \%$ (95\% CI: 72-91\%), Table 2 and Figs. 2a-g.

Heterogeneity between studies evaluating normal anal samples was $\mathrm{I}^{2}=0 \%(95 \% \mathrm{CI}: 0-13 \%)$, in AIN1/LSIL (excluding condyloma) $\mathrm{I}^{2}=71 \%$ (95\% CI: $39-86 \%$ ), in all LSIL (including condyloma) $\mathrm{I}^{2}=59 \%$ (95\% CI: $23-78 \%$ ), AIN2 $\mathrm{I}^{2}=77 \%$ (95\% CI: $\left.55-88 \%\right)$, AIN3 $\mathrm{I}^{2}=15 \%(95 \%$ CI: $0-79 \%)$, HSIL in a two-tiered nomenclature $\mathrm{I}^{2}=85 \%$ (95\% CI: 71-92\%), and for all combined results from HSIL $\mathrm{I}^{2}=84 \%$ (95\% CI: 76-90\%) (Table 2). Concerning publication bias, funnel plots were derived (supplemental digital content Supplementary Figures 1A-1G).

\section{Discussion}

Different definitions for a positive p16 expression have been used frequently in the literature. According to the LAST recommendations [3], a positive result should be considered when there is a diffuse block staining with strong nuclear or nuclear plus cytoplasmic staining. Ideally the staining should be in the basal cell layer with extension upward involving at least one-third of the epithelial thickness, but this is not a strict criterion for a positive result. Focal or patchy nuclear staining and exclusive cytoplasmic staining should be interpreted as negative [3]. To ensure that the more accurate results were obtained, only studies that considered positivity according to the LAST definition [3] were included in our meta-analysis.

In the new two-tiered nomenclature proposed by LAST, the LSIL category merged LSIL/-IN1 and condyloma into a single low-grade category. Subsequently, it is important, to present $\mathrm{p} 16$ positivity in all anal LSIL samples, according to the definition proposed and currently more accepted. However, condylomas in different anogenital sites, like the cervix vs. anus, have a different prevalence (rare in the cervix) and, the relative frequency of -IN1 vs. condyloma in a given site can have a major impact on the p16 expression rate of low-grade lesions in that site. Taking this into consideration, p16 positivity was also calculated in a category denominated AIN1/LSIL, excluding condylomas, allowing a better comparison between the anus and the cervix. The calculation in condylomas was not performed due to the low number of "isolated" anal condylomas samples and studies, in which p16 was performed/reported.

As far as we know, only one meta-analysis was published on p16 immunostaining in HPV-associated squamous lesions of the lower anogenital tract. This analysis was exclusively on cervical samples [4] and was conducted before the publication of the LAST classification [3]. It 
Fig. 1 : Study selection

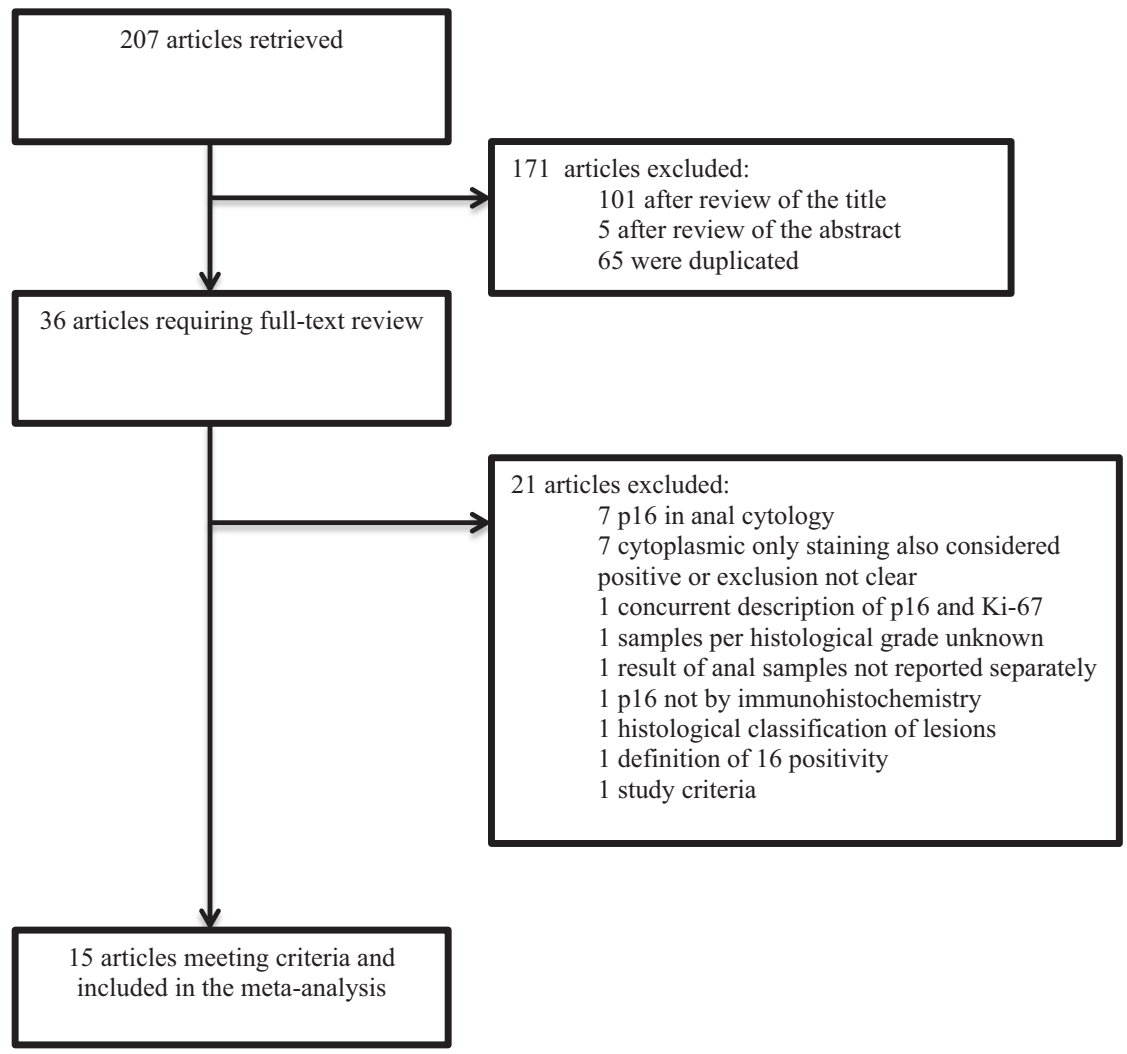

included 61 studies and evaluated cytological and histological specimens from the uterine cervix. There was a positive association with the increasing severity of cytological and histologic abnormalities and correspondingly higher rates of p16-positive staining. In histology, $2 \%$ of normal biopsies (95\% CI: $0.4-30 \%), 38 \%$ of CIN1 $(95 \%$ CI: $23-53 \%), 68 \%$ of CIN2 (95\% CI: $44-92 \%$ ), and $82 \%$ of CIN3 (95\% CI: 72-92\%) were positive. In this study, authors defined a positive result in histological samples as a diffuse staining ( $>25 \%$ of cells stained). No description of nuclear and/or cytoplasmic positivity was given. p16 expression in CIN1 and CIN2 revealed extreme inter-study variation, with lower variation in normal and CIN3 lesions. When compared with our meta-analysis, results in normal samples ( $2 \%$ vs. $2 \%$ ), AIN2/CIN2 (76\% vs. $68 \%$ ), and AIN3/CIN3 (90\% vs. $82 \%$ ) were in the similar range. However, for AIN1/LSIL (excluding condylomas) and CIN1, the results were very different with positive rates of $12 \%$ in the anus and $38 \%$ in the cervix. The LAST consensus [3] reported that the expected positive rates to be 80-90\% in -IN2 and approximately $99 \%$ in -IN3 cases in lower anogenital tract squamous lesions. For CIN1, at least $30 \%$ of the cases are expected to be p16 positive [3] and similar conclusions are commonly drawn for other anogenital sites including anal LSIL/AIN1 samples [28]. This difference in positive rates for p16 between low-grade cervical and anal lesions could be related to the heterogeneity in the definition of a positive result used in studies relating to cervical disease and/or the different definitions for positivity in the two meta-analyses, but, a different mechanism cannot be excluded. Our results showed that p16 positivity in an anal low-grade category that includes condyloma is lower than an anal low-grade category without condylomas ( $7 \%$ vs. $12 \%$, respectively). This expression is still lower than what is generally described in the cervix and, in the previous cervical metaanalysis (38\%). Although, the inclusion of condylomas has an impact on the positivity in anal low-grade lesions, this does not seem to explain the difference in the rates between low-grade lesions of the anus and the cervix. Differentiating the results from the condylomas and AIN1/LSIL (without condylomas) categories poses some challenges. There is a possibility that condylomas were not recognized or distinguished from AIN1 in some of the studies included in the AIN1/LSIL category. Many of the conclusions for HPVrelated lower anogenital tract neoplasia management, including p16 immunostaining are considered applicable across all anogenital sites, mostly based on generalizations from the cervix [3]. This may not to be the case for $\mathrm{p} 16$ expression in low-grade ASILs. Although, the several similarities between anal and cervical carcinogenesis, large differences certainly exist, new cases of cervical cancers diagnosed yearly worldwide are much higher than anal cancer [29]. 


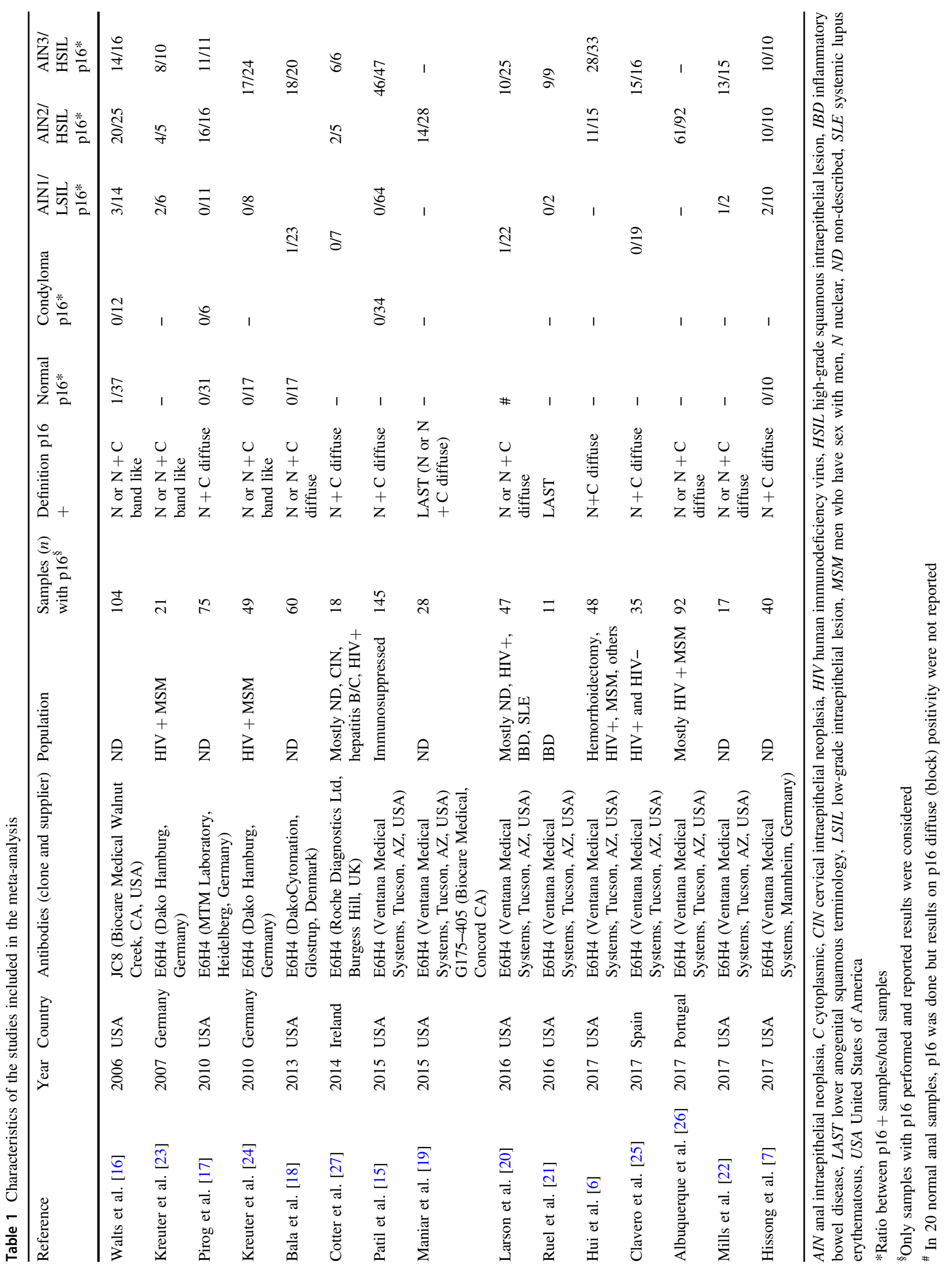


Table 2 Meta-analysis of the p16 proportion and test for heterogeneity stratified by histologic grade

\begin{tabular}{|c|c|c|c|c|c|c|}
\hline Study & \# Total & Positive samples & $\%$ & $95 \% \mathrm{CI}$ & Weight $(\%)$ & $\mathrm{I}^{2}(95 \% \mathrm{CI})$ \\
\hline \multicolumn{7}{|c|}{ Meta-analysis: proportion-normal samples } \\
\hline Walts, 2006 & 37 & 1 & 2.703 & $0.0684-14.160$ & 32.48 & $0 \%(0-13 \%)$ \\
\hline Pirog, 2010 & 31 & 0 & 0.000 & $0.000-11.219$ & 27.35 & \\
\hline Kreuter, 2010 & 17 & 0 & 0.000 & $0.000-19.506$ & 15.38 & \\
\hline Bala, 2013 & 17 & 0 & 0.000 & $0.000-19.506$ & 15.38 & \\
\hline Hissong, 2017 & 10 & 0 & 0.000 & $0.000-30.850$ & 9.40 & \\
\hline $\begin{array}{l}\text { Total (random } \\
\text { effects) }\end{array}$ & 112 & 1 & 1.930 & $0.238-5.196$ & 100 & \\
\hline \multicolumn{7}{|c|}{ Meta-analysis: proportion-AIN1/ LSIL (excluding condylomas) } \\
\hline Walts, 2006 & 14 & 3 & 21.429 & $4.658-50.798$ & 12.00 & $71 \%(39-86 \%)$ \\
\hline Kreuter, 2007 & 6 & 2 & 33.333 & $4.327-77.722$ & 5.60 & \\
\hline Pirog, 2010 & 11 & 0 & 0.000 & $0.000-28.491$ & 9.60 & \\
\hline Kreuter, 2010 & 8 & 0 & 0.000 & $0.000-36.942$ & 7.20 & \\
\hline Patil, 2015 & 64 & 0 & 0.000 & $0.000-5.601$ & 52.00 & \\
\hline Ruel, 2016 & 2 & 0 & 0.000 & $0.000-84.189$ & 2.40 & \\
\hline Mills, 2017 & 2 & 1 & 50.000 & $1.258-98.742$ & 2.40 & \\
\hline Hissong, 2017 & 10 & 2 & 20.000 & $2.521-55.610$ & 8.80 & \\
\hline $\begin{array}{l}\text { Total (random } \\
\text { effects) }\end{array}$ & 117 & 8 & 11.767 & $2.436-26.748$ & 100 & \\
\hline \multicolumn{7}{|c|}{ Meta-analysis: proportion-LSIL (all classified as AIN1/LSIL/condyloma) } \\
\hline Walts, 2006 & 26 & 3 & 11.538 & $2.446-30.154$ & 11.21 & $59 \%(23-78 \%)$ \\
\hline Kreuter, 2007 & 6 & 2 & 33.333 & $4.327-77.722$ & 5.86 & \\
\hline Pirog, 2010 & 17 & 0 & 0.000 & $0.000-19.506$ & 9.67 & \\
\hline Kreuter, 2010 & 8 & 0 & 0.000 & $0.000-36.942$ & 6.84 & \\
\hline Bala, 2013 & 23 & 1 & 4.348 & $0.110-21.949$ & 10.78 & \\
\hline Cotter, 2014 & 7 & 0 & 0.000 & $0.000-40.962$ & 6.37 & \\
\hline Patil, 2015 & 98 & 0 & 0.000 & $0.000-3.694$ & 14.62 & \\
\hline Larson, 2016 & 22 & 1 & 4.545 & $0.115-22.844$ & 10.62 & \\
\hline Ruel, 2016 & 2 & 0 & 0.000 & $0.000-84.189$ & 3.15 & \\
\hline Clavero, 2017 & 19 & 0 & 0.000 & $0.000-17.647$ & 10.08 & \\
\hline Mills, 2017 & 2 & 1 & 50.000 & $1.258-98.742$ & 3.15 & \\
\hline Hissong, 2017 & 10 & 2 & 20.000 & $2.521-55.610$ & 7.65 & \\
\hline $\begin{array}{l}\text { Total (random } \\
\text { effects) }\end{array}$ & 240 & 10 & 6.520 & $2.133-13.063$ & 100 & \\
\hline \multicolumn{7}{|c|}{ Meta-analysis: proportion-AIN2 } \\
\hline Walts, 2006 & 25 & 20 & 80.000 & $59.296-93.169$ & 14.33 & $77 \%(55-88 \%)$ \\
\hline Kreuter, 2007 & 5 & 4 & 80.000 & $28.358-99.495$ & 8.65 & \\
\hline Pirog, 2010 & 16 & 16 & 100.000 & $79.409-100.000$ & 12.98 & \\
\hline Cotter, 2014 & 5 & 2 & 40.000 & $5.274-85.337$ & 8.65 & \\
\hline Maniar, 2015 & 28 & 14 & 50.000 & $30.647-69.353$ & 14.63 & \\
\hline Hui, 2017 & 15 & 11 & 73.333 & $44.900-92.213$ & 12.76 & \\
\hline Albuquerque, 2017 & 92 & 61 & 66.304 & $55.696-75.830$ & 16.70 & \\
\hline Hissong, 2017 & 10 & 10 & 100.000 & $69.150-100.000$ & 11.30 & \\
\hline $\begin{array}{l}\text { Total (random } \\
\text { effects) }\end{array}$ & 196 & 138 & 76.029 & $60.885-88.396$ & 100 & \\
\hline \multicolumn{7}{|c|}{ Meta-analysis: proportion—AIN3 } \\
\hline Walts, 2006 & 16 & 14 & 87.500 & $61.652-98.449$ & 18.93 & $15 \%(0-79 \%)$ \\
\hline Kreuter, 2007 & 10 & 8 & 80.000 & 44.390-97.479 & 13.06 & \\
\hline
\end{tabular}


Table 2 (continued)

\begin{tabular}{|c|c|c|c|c|c|c|}
\hline Study & \# Total & Positive samples & $\%$ & $95 \% \mathrm{CI}$ & Weight (\%) & $\mathrm{I}^{2}(95 \% \mathrm{CI})$ \\
\hline Pirog, 2010 & 11 & 11 & 100.000 & $71.509-100.000$ & 14.09 & \\
\hline Cotter, 2014 & 6 & 6 & 100.000 & $54.074-100.000$ & 8.70 & \\
\hline Hui, 2017 & 33 & 28 & 84.848 & $68.101-94.891$ & 32.16 & \\
\hline Hissong, 2017 & 10 & 10 & 100.000 & $69.150-100.000$ & 13.06 & \\
\hline $\begin{array}{l}\text { Total (random } \\
\text { effects) }\end{array}$ & 86 & 77 & 89.542 & $81.605-95.434$ & 100 & \\
\hline \multicolumn{7}{|c|}{ Meta-analysis: proportion-HSIL } \\
\hline Kreuter, 2010 & 24 & 17 & 70.833 & $48.905-87.385$ & 14.81 & $85 \%(71-92 \%)$ \\
\hline Bala, 2013 & 20 & 18 & 90.000 & $68.302-98.765$ & 14.44 & \\
\hline Patil, 2015 & 47 & 46 & 97.872 & $88.706-99.946$ & 15.84 & \\
\hline Larson, 2016 & 25 & 10 & 40.000 & $21.125-61.335$ & 14.89 & \\
\hline Ruel, 2016 & 9 & 9 & 100.000 & $66.373-100.000$ & 12.31 & \\
\hline Clavero, 2017 & 16 & 15 & 93.750 & $69.768-99.842$ & 13.93 & \\
\hline Mills, 2017 & 15 & 13 & 86.667 & $59.540-98.342$ & 13.77 & \\
\hline $\begin{array}{l}\text { Total (random } \\
\text { effects) }\end{array}$ & 156 & 128 & 83.922 & $66.467-95.753$ & 100 & \\
\hline \multicolumn{7}{|c|}{ Meta-analysis: proportion-HSIL (all classified as AIN2, AIN3, or HSIL) } \\
\hline Walts, 2006 & 41 & 34 & 82.927 & 67.944-92.848 & 7.29 & $84 \%(76-90 \%)$ \\
\hline Kreuter, 2007 & 15 & 12 & 80.000 & $51.911-95.669$ & 6.16 & \\
\hline Pirog, 2010 & 27 & 27 & 100.000 & $87.230-100.000$ & 6.90 & \\
\hline Kreuter, 2010 & 24 & 17 & 70.833 & $48.905-87.385$ & 6.77 & \\
\hline Bala, 2013 & 20 & 18 & 90.000 & $68.302-98.765$ & 6.55 & \\
\hline Cotter, 2014 & 11 & 8 & 72.727 & $39.026-93.978$ & 5.68 & \\
\hline Patil, 2015 & 47 & 46 & 97.872 & $88.706-99.946$ & 7.40 & \\
\hline Maniar, 2015 & 28 & 14 & 50.000 & $30.647-69.353$ & 6.94 & \\
\hline Larson, 2016 & 25 & 10 & 40.000 & $21.125-61.335$ & 6.82 & \\
\hline Ruel, 2016 & 9 & 9 & 100.000 & $66.373-100.000$ & 5.35 & \\
\hline Hui, 2017 & 48 & 39 & 81.250 & $67.371-91.050$ & 7.41 & \\
\hline Clavero, 2017 & 16 & 15 & 93.750 & $69.768-99.842$ & 6.25 & \\
\hline Albuquerque, 2017 & 92 & 61 & 66.304 & $55.696-75.830$ & 7.78 & \\
\hline Mills, 2017 & 15 & 13 & 86.667 & $59.540-98.342$ & 6.16 & \\
\hline Hissong, 2017 & 20 & 20 & 100.000 & $83.157-100.000$ & 6.55 & \\
\hline $\begin{array}{l}\text { Total (random } \\
\text { effects) }\end{array}$ & 438 & 343 & 82.483 & $72.412-90.655$ & 100 & \\
\hline
\end{tabular}

$A I N$ anal intraepithelial neoplasia, CI confidence interval, HSIL high-grade squamous intraepithelial lesions, LSIL low-grade squamous intraepithelial lesions

A major indication for p16 immunostaining is in cases of -IN2, including AIN2, as there is greater variability in reporting among pathologists and an objective resolution regarding HSIL/LSIL classification is needed for clinical management. There is no recommendation for p16 immunostaining use in -IN3 [3] and, such use may potentially lead to the exclusion of p16-negative samples from treatment and, consequently, leading to under-treatment. Falsepositive results leading to over-treatment is also an important point to consider, through upgrading lesions with p16 positivity that would have been diagnosed as low-grade without this staining $[3,26]$. This is minimized by the restricted recommendation of using p16 in $\leq-\mathrm{IN} 1$ (biopsy specimens interpreted as $\leq-\mathrm{IN} 1$ that are at high risk for missed high-grade disease) [3].

p16 expression in anal squamous cell carcinoma was not included in this study because this staining is not currently recommended for anogenital cancer and also due to the low number of anal squamous cell carcinoma samples tested in the studies included. Three studies $[6,18,21]$ in this metaanalysis performed p16 immunostaining in anal squamous cell carcinoma, with a combined total of 28 samples. 
a

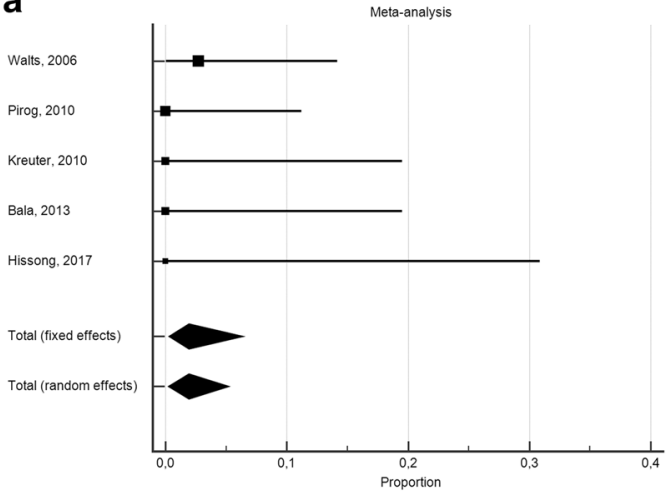

C

Wats, 2006 Kreuter, 2007 Pirog, 2010 Kreuter, 2010

Bala, 2013

Cotter, 2014

Patil, 2015

Larson, 2016

Ruel. 2016

Clavero, 2017

Mills, 2017

Hissong, 2017

Total (fixed effects)

Total (random effects)

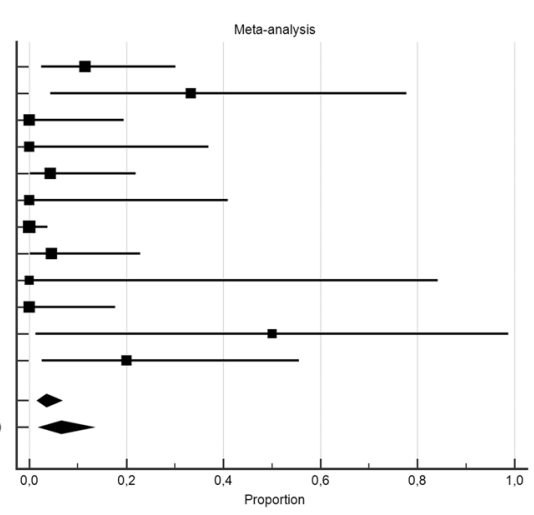

e

Walts, 2006

Kreuter, 2007

Pirog, 2010

Cotter, 2014

Hui. 2017

Hissong, 2017

Total (fixed effects)

Total (random effects)

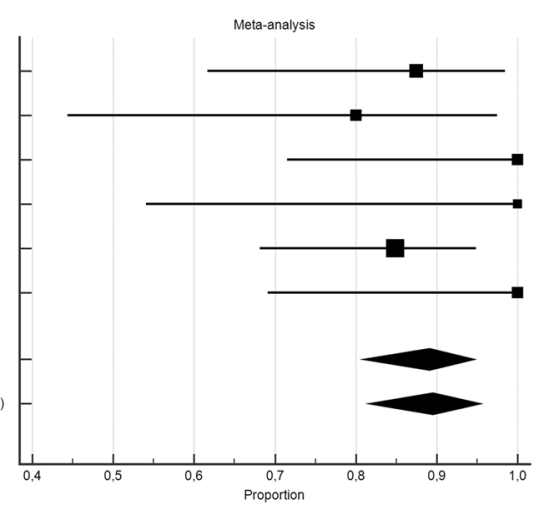

g

Walts, 2006
Kreuter, 2007

Prog, 2010

Kreuter, 2010
Bala, 2013

Coter 2014

Patil, 2015

Maniar. 2015

LArson, 2016

Ruel, 2016

Hui, 2017

Clavero. 2017

Albuquerque, 2017

Mills, 2017

Hissong. 2017

Total (fixed effects)

Total (random effects)

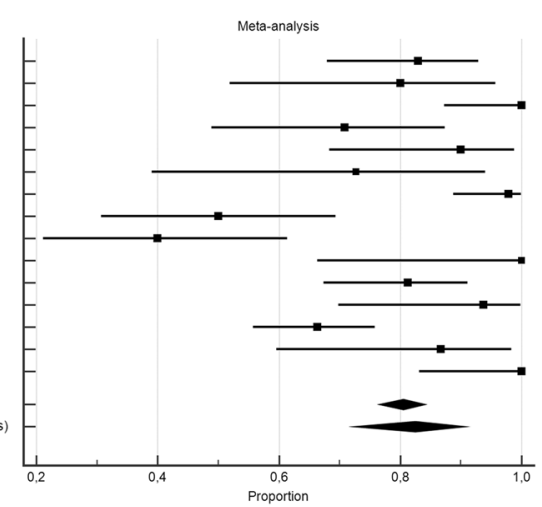

b

Walls, 2006

Kreuter, 2007

Pirog. 2010

Kreuter, 2010

Patil, 2015

Ruel, 2016

Mills, 2017

Hissong, 2017

Total (fixed effects)

Total (random effects)

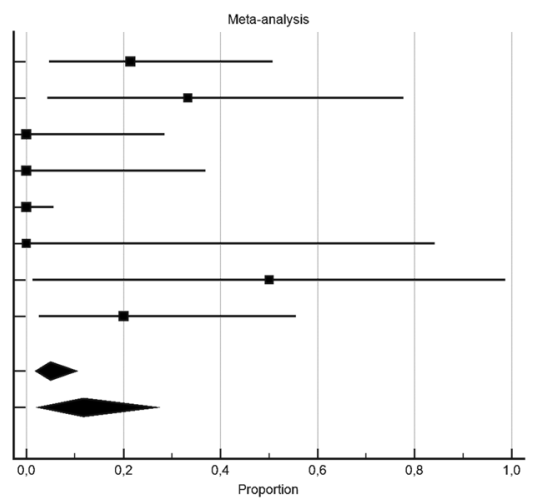

d

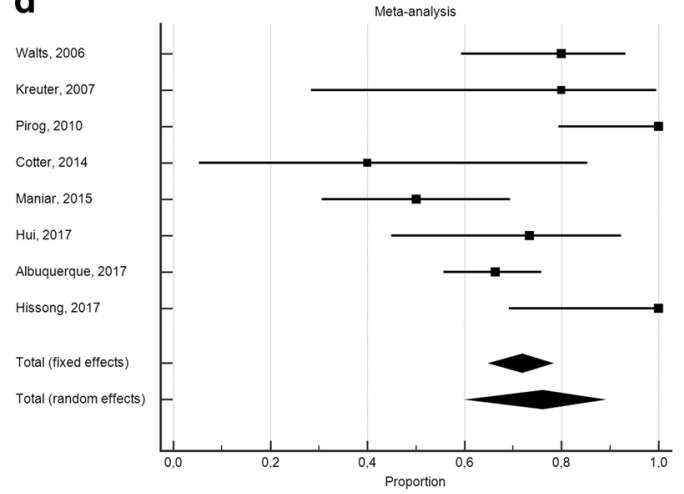

f

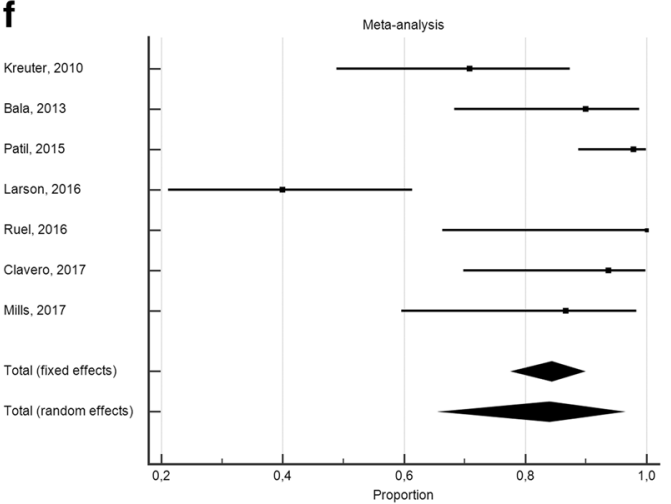


Fig. 2 a Meta-analysis of the normal samples stained positive for $\mathrm{p} 16$. b Meta-analysis of the AIN1/LSIL (excluding condylomas) stained positive for p16. c Meta-analysis of the LSIL samples (AIN1/LSIL/ condyloma) stained positive for p16. d Meta-analysis of the AIN2 samples stained positive for p16. e Meta-analysis of the AIN3 samples stained positive for p16. f Meta-analysis of the HSIL samples (two-tiered nomenclature) stained positive for p16. g Metaanalysis of all of the combined HSIL (AIN2, AIN3, and HSIL) samples stained positive for $\mathrm{p} 16$

As for the previously conducted meta-analysis of cervical disease, there is substantial heterogeneity in the studies included in this meta-analysis, especially, in cases of AIN1/ LSIL, AIN2, and HSIL. There was no/low heterogeneity between studies on the evaluation of normal anal samples and AIN3.

Some studies in cervical disease have evaluated the possible role of p16 in determining LSIL progression. Some of these studies [30-34] showed that low-grade cervical lesions (CIN1) that were p16 positive progressed more frequently to high-grade lesions (CIN2/CIN3) compared with p16-negative CIN1, although there are also other studies in which this association was not found [35, 36]. Currently, there are no formal recommendations for LSIL management based on p16 results [3]. In low-grade cervical lesions, false-positive results are common and the lower positive predictive value may limit the role of p16 as a prognostic marker [37]. In low-grade anal lesions, false positives are uncommon in most of the studies, so this may not be the same case for prognostic value in ASIL. Currently, there are no studies assessing p16 as a prognostic marker in anal LSIL. There is, however, information on the clinical impact of downgrading -IN2/AIN2 samples and the prognosis of -IN2/AIN2 samples based on p16 immunostaining. Albuquerque et al. [26] showed that $34 \%$ of AIN2 are p16 negative, while Maniar et al. [19] found $50 \%$ of AIN2 to be p16 negative in their respective populations. Albuquerque et al. [26] study further demonstrated that the AIN2 p16-negative samples showed lower rates of progression in the follow-up with high-resolution anoscopy. The possible prognostic value, especially in anal LSIL, needs to be explored in future studies.

\section{Conclusions}

The current information and recommendations for HPVassociated squamous intraepithelial lesions of the lower anogenital tract come almost exclusively from cervical studies and this stands true also for p16 immunostaining. It is fundamental to ensure that data on biomarkers in anal cancer and anal precancerous lesions are based on specific scientific evidence from this anatomical area and not just translated from cervical carcinogenesis. p16 positivity in ASIL appears to be lower for anal low-grade lesions compared with that commonly described in cervical low-grade lesions.

\section{Compliance with ethical standards}

Conflict of interest The authors declare that they have no conflict of interest.

\section{References}

1. Maniar KP, Nayar R. HPV-related squamous neoplasia of the lower anogenital tract: an update and review of recent guidelines. Adv Anat Pathol. 2014;21:341-58.

2. Mahajan A. Practical issues in the application of p16 immunohistochemistry in diagnostic pathology. Hum Pathol. 2016;51:64-74.

3. Darragh TM, Colgan TJ, Cox JT, et al. The lower anogenital squamous terminology standardization project for HPV-associated lesions: background and consensus recommendations from the College of American Pathologists and the American Society for Colposcopy and Cervical Pathology. J Low Genit Tract Dis. 2012;16:205-42. Review. Erratum in: J Low Genit Tract Dis. 2013;17:368

4. Tsoumpou I, Arbyn M, Kyrgiou M, et al. p16 (INK4a) immunostaining in cytological and histological specimens from the uterine cervix: a systematic review and meta-analysis. Cancer Treat Rev. 2009;35:210-20.

5. Smittenaar CR, Petersen KA, Stewart K, et al. Cancer incidence and mortality projections in the UK until 2035. Br J Cancer. 2016;115:1147-55.

6. Hui Y, Quddus MR, Murthy JN, et al. Human papillomavirus genotyping of incidental malignant and premalignant lesions on hemorrhoidectomy specimens. Am J Surg Pathol. 2017;41:382-8.

7. Hissong E, Cimic A, Leeman A, et al. Utility of stathmin-1 as a novel marker in evaluating anal intraepithelial neoplasia (AIN). Appl Immunohistochem Mol Morphol. 2017. https://doi.org/ 10.1097/PAI.0000000000000547.

8. Bean SM, Eltoum I, Horton DK, et al. Immunohistochemical expression of p16 and $\mathrm{Ki}-67$ correlates with degree of anal intraepithelial neoplasia. Am J Surg Pathol. 2007;31:555-61.

9. Bean SM, Meara RS, Vollmer RT, et al. p16 Improves interobserver agreement in diagnosis of anal intraepithelial neoplasia. $\mathrm{J}$ Low Genit Tract Dis. 2009;13:145-53.

10. Kreuter A, Siorokos C, Oellig F, et al. High-grade dysplasia in anogenital warts of HIV-positive men. JAMA Dermatol. 2016;152:1225-30.

11. Siegenbeek van Heukelom ML, Richel O, de Vries HJ, et al. Low and high-risk human papillomavirus genotype infections in intraanal warts in HIV-positive men who have sex with men. Br J Dermatol. 2016;175:735-43.

12. Walts AE, Lechago J, Hu B, et al. P16 and Ki67 immunostains decrease intra and interobserver variability in the diagnosis and grading of anal intraepithelial neoplasia (AIN). Clin Med Pathol. 2008;1:7-13.

13. Bernard JE, Butler MO, Sandweiss L, et al. Anal intraepithelial neoplasia: correlation of grade with p16INK4a immunohistochemistry and HPV in situ hybridization. Appl Immunohistochem Mol Morphol. 2008;16:215-20.

14. Walker F, Abramowitz L, Benabderrahmane D, et al. Growth factor receptor expression in anal squamous lesions: modifications 
associated with oncogenic human papillomavirus and human immunodeficiency virus. Hum Pathol. 2009;40:1517-27.

15. Patil DT, Yang B. Utility of human papillomavirus capsid protein L1 and p16 in the assessment and accurate classification of anal squamous intraepithelial lesions. Am J Clin Pathol. 2015;144:113-21.

16. Walts AE, Lechago J, Bose S. p16 and Ki67 immunostaining is a useful adjunct in the assessment of biopsies for HPV-associated anal intraepithelial neoplasia. Am J Surg Pathol. 2006;30:795-801.

17. Pirog EC, Quint KD, Yantiss RK. P16/CDKN2A and Ki-67 enhance the detection of anal intraepithelial neoplasia and condyloma and correlate with human papillomavirus detection by polymerase chain reaction. Am J Surg Pathol. 2010;34:1449-55.

18. Bala R, Pinsky BA, Beck AH, et al. p16 is superior to ProEx C in identifying high-grade squamous intraepithelial lesions (HSIL) of the anal canal. Am J Surg Pathol. 2013;37:659-68.

19. Maniar KP, Sanchez B, Paintal A, et al. Role of the biomarker p16 in downgrading -IN2 diagnoses and predicting higher-grade lesions. Am J Surg Pathol. 2015;39:1708-18.

20. Larson BK, Mohanty SK, Wu JM, et al. ProEx ${ }^{\mathrm{TM}} \mathrm{C}$ is a useful ancillary study for grading anal intraepithelial neoplasia alone and in combination with other biomarkers. Acta Pathol Microbiol Immunol Scand. 2016;124:175-80.

21. Ruel J, Ko HM, Roda G, et al. Anal neoplasia in inflammatory bowel disease is associated with HPV and perianal disease. Clin Transl Gastroenterol. 2016;7:e148.

22. Mills AM, Dirks DC, Poulter MD, et al. HR-HPV E6/E7 mRNA in situ hybridization: validation against PCR, DNA in situ hybridization, and p16 immunohistochemistry in 102 Samples of cervical, vulvar, anal, and head and neck neoplasia. Am J Surg Pathol. 2017;41:607-15.

23. Kreuter A, Wieland U, Gambichler T, et al. p16ink4a expression decreases during imiquimod treatment of anal intraepithelial neoplasia in human immunodeficiency virus-infected men and correlates with the decline of lesional high-risk human papillomavirus DNA load. Br J Dermatol. 2007;157:523-30.

24. Kreuter A, Jesse M, Potthoff A, et al. Expression of proliferative biomarkers in anal intraepithelial neoplasia of HIV-positive men. J Am Acad Dermatol. 2010;63:490-8.

25. Clavero O, McCloskey JC, Molina VM, et al. Squamous intraepithelial lesions of the anal squamocolumnar junction: histopathological classification and HPV genotyping. Papillomavirus Res. 2017;3:11-17.

26. Albuquerque A, Rios E, Macedo G. The impact of p16 immunostaining in reducing anal squamous intraepithelial lesions indication for treatment. Am J Surg Pathol. 2017;41:1151-2.

27. Cotter MB, Kelly ME, O'Connell PR, et al. Anal intraepithelial neoplasia: a single centre 19 year review. Colorectal Dis. 2014; $16: 777-82$

28. Ma C, Pai RK. Predictive value of immunohistochemistry in premalignant lesions of the gastrointestinal tract. Semin Diagn Pathol. 2015;32:334-43.

29. Yang EJ, Quick MC, Hanamornroongruang S, et al. Microanatomy of the cervical and anorectal squamocolumnar junctions: a proposed model for anatomical differences in HPV-related cancer risk. Mod Pathol. 2015;28:994-1000.

30. Negri G, Vittadello F, Romano F, et al. p16INK4a expression and progression risk of low-grade intraepithelial neoplasia of the cervix uteri. Virchows Arch. 2004;445:616-20.

31. Ozaki S, Zen $\mathrm{Y}$, Inoue $\mathrm{M}$. Biomarker expression in cervical intraepithelial neoplasia: potential progression predictive factors for low-grade lesions. Hum Pathol. 2011;42:1007-12.

32. Del Pino M, Garcia S, Fuste V, et al. Value of p16 (INK4a) as a marker of progression/ regression in cervical intraepithelial neoplasia grade 1. Am J Obstet Gynecol. 2009;201:488.e1-7.

33. Cortecchia S, Galanti G, Sgadari C, et al. Follow-up study of patients with cervical intraepithelial neoplasia grade 1 overexpressing p16Ink4a. Int J Gynecol Cancer. 2013;23:1663-9.

34. Liao GD, Sellors JW, Sun HK, et al. p16INK4 A immunohistochemical staining and predictive value for progression of cervical intraepithelial neoplasiagrade1: a prospective study in China. Int J Cancer. 2014;134:1715-24.

35. Quint KD, de Koning MN, Quint WG, et al. Progression of cervical low-grade squamous intraepithelial lesions: in search of prognostic biomarkers. Eur J Obstet Gynecol Reprod Biol. 2013;2115:319-29.

36. Mills AM, Paquette C, Castle PE, et al. Risk stratification by p16 immunostaining of CIN1 biopsies: a retrospective study of patients from the quadrivalent HPV vaccine trials. Am J Surg Pathol. 2015;3:611-7.

37. Pirog E. Immunohistochemistry and in situ hybridization for the diagnosis and classification of squamous lesions of the anogenital region. Semin Diagn Pathol. 2015;32:409-18. 